\title{
Implementation of Crop Simulation Model for Malwa Region Punjab
}

\author{
Parmeet Kaur $^{1}$, Er. Sikander Singh Cheema ${ }^{2}$ \\ Research Student, M. Tech. (CE), Department of Computer Engineering, Punjabi University, Patiala ${ }^{1}$ \\ Assistant Professor, Department of Computer Engineering, Punjabi University, Patiala ${ }^{2}$
}

\begin{abstract}
Crop Simulation Models helps in precision decisions in agriculture that are used to integrate various datasets through information technology in building crop production decisions. Crop Diversification is also necessary to properly utilize land resources and for increasing crop production. This model works on the idea to estimate crop production on a particular area and gives us simulated crop production on that area in specific soil and weather conditions. We have compared the results of simulation production and actual production to check the validity of the simulation model. It gives satisfactory results. This model also gives suggestions for crop diversification as it is necessary to increase income of farmers and for increasing fertility of the land. It also tells us to select appropriate crops according to the conditions of the soil. This model is specifically designed for Malwa Region, Punjab and works according to the climatic conditions of this particular area.
\end{abstract}

Keywords: Agriculture, crop, diversification, simulation, soil, weather.

\section{INTRODUCTION}

Agriculture is the major occupation required to fulfil basic needs of human life that are food and clothes. The Food and Agriculture Organization (FAO) has suggested that the annual production of crops should be increased by 60 percent than it was in 2006, to meet the food requirements in 2050. The agriculture and forestry generate one- fifth of the green house gas emissions. As agriculture is important aspect in food security, it should be well maintained and should have high production to meet future needs of people with increasing population.[1] The people who are involved in agriculture should have proper knowledge about all its instruments and digital techniques, so that they could take advantage of the modern advancement. But as we talk about the agriculture in Punjab, it is not that much advanced. Mostly of the people are not aware of new technologies and are using traditional techniques to grow various crops. It may result into loss or profit depending on the luck and weather conditions. That's why the condition of famer is not very strong. Whenever there is a loss in the production of crops due to the mono-crop culture or any other reason like weather conditions, it leads to disappointment. Due to this reason the suicides by the farmers are increasing day by day. [2]

The solution of this problem is to give knowledge to the farmers about the various techniques and let them know whether their land and weather is suitable for particular crop or not. We have developed a Crop Simulation Model for this purpose and particularly for Malwa Region, Punjab. This model is designed to simulate the influence of crop diversification, crop rotation and soil and weather parameters on the particular crop.

We should focus on crop diversification in Punjab because it has become wheat and paddy prone cultivation area. According to the Agricultural Census of India, the area of Punjab which was the under wheat and paddy was $81.5 \%$ during Rabi and Kharif season.[3] It leads to various problems such as due to free supply of water and electricity there is a complete wastage of these resources. The other problems due to such rotation are infertility of soil, loss of ground water gradually. People don't go for crop diversification due to some risks because all the farmers in Punjab are depending on the agriculture for their income. These risks include price risk, labour risk, credit risk and yield risk. [4]

\section{OBJECTIVES}

A system is a well defined structure which contains many interrelated elements that can interact with each other. A system can be dynamic when it changes its behaviour over time. A model is defined as a simplified representation of the system and a simulation can be considered as study of the system and its behaviour using models. [5]

This model is for Malwa region which is located in west-central India and has sub-tropic and semi-arid climate and most of the land contains plains. The main weather input requirements for the model are maximum and minimum temperature and rainfall quantity to make balance of requirements of water by the plants. The main soil requirements for the model are $\mathrm{pH}$ of the soil, soil texture that contains content of sand, slit and clay in the soil and electrical conductivity of the soil. 
This model will have two working modules that will work independently. These can be defined as follows:

\section{A. Crop simulation model}

Crop Simulation is defined as the estimation of production of crops before the actual growth of the crops. It checks crop production with the help of various models related to it and parameter files that are attached to it as database. This Crop Simulation Model can do following functions:

- It gives results about various crops and their required soil and weather conditions.

- It can give us the name of crop suitable for that soil, when we give input as soil and weather conditions.

- It tells about the production of various soils according to conditions on a particular area.

- It suggests about crop diversification and suitable crops in that area that can be added in rotation.[6]

\section{B. Crop diversification}

Crop Diversification can be defined as growing various crops on a particular land in rotation to maintain the fertility of soil. It helps to conserve water and other resources and also results in increase in production and income of the farmer if the selection of the crops would be appropriate and according to the specifications of the land and weather. In our model, we only suggest Crop Diversification and various alternative crops that can be used for crop diversification and drawing the data from existing models. [7]

Diversification can be verified or analyzed by dividing the land into various parts that can be marginal, medium and large. Farmers can increase their income if they go for crop diversification and try to cultivate various crops in rotation. But it also comprises of the fact that the crops chosen for the rotation should be properly selected so that they come up with the best results. This model will give the information about the crops that are suitable for the land in that area.[8]

\section{METHODOLOGY}

A crop model is designed to diagnose the uncertainties in the production and growth of various crops due to the reasons like crop type, soil type, weather and other parameters. The main function of this model is to check for the suitable weather conditions and soil conditions for a particular crop and suggest to grow only that particular crop on that area to yield maximum benefit. It will also help us to check the results of crop diversification and helps to maintain soil nutrients. [9]

Crop Diversification can provide us a varied amount of food under varied weather conditions,

which will result into better production. The average annual growth rate of food grain production has declined from 3.22 to 1.66 from the years 1950s to 1990s. This leads to the decline in per capita income and growth of the country or particular area. Thus crop diversification is suggested to handle this decline.[10] Crop Diversification can be applied in two conditions: first one is when to develop access to irrigation that is we grow such crops that are prone to water demands, and the second one is when there is a lack of irrigation due to adverse weather conditions. [11]

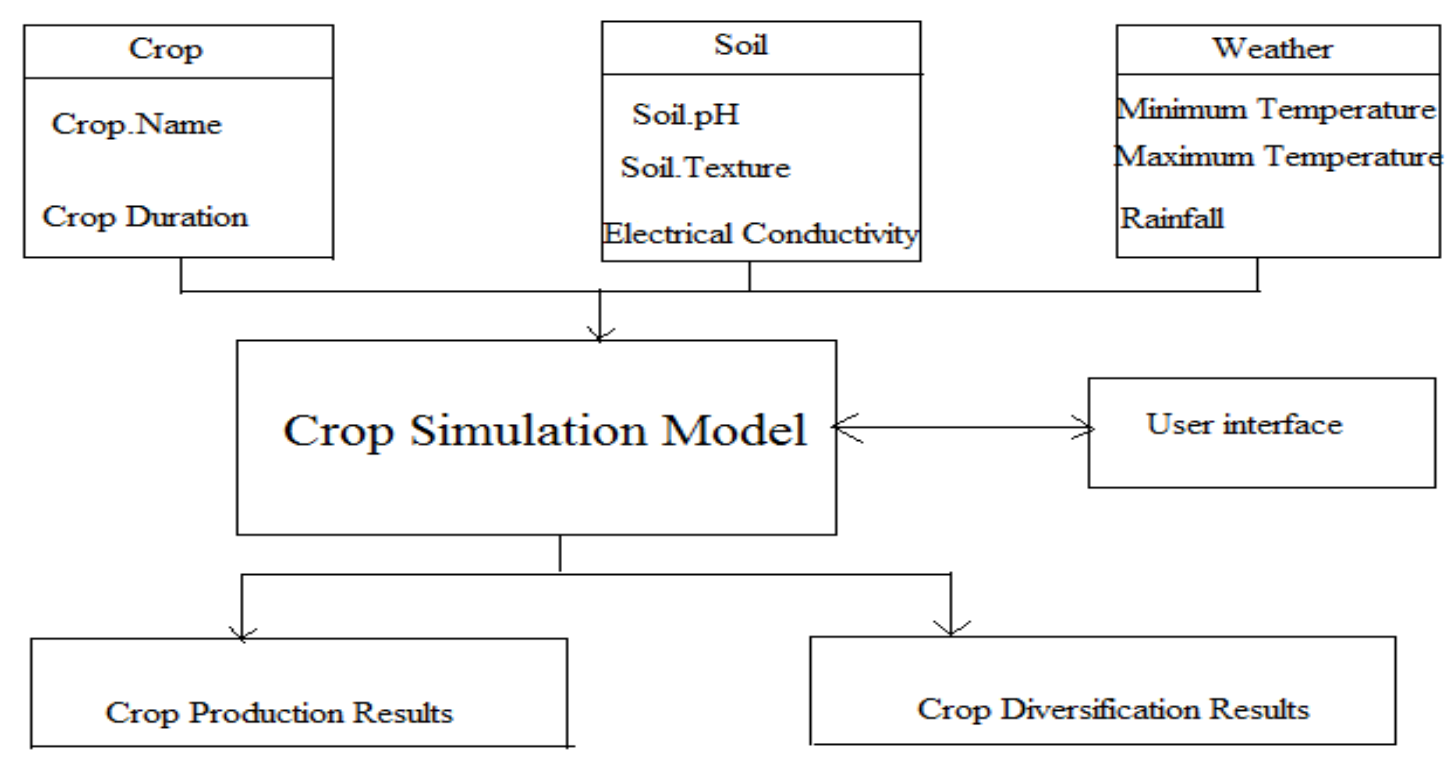

Figure.1- Working Strategy of Crop Simulation Model 
The inputs to the system of crop simulation model can be defined as following :-

1. Soil Inputs: It contains the $\mathrm{pH}$ value of the soil, electrical conductivity of the soil, Soil texture of that land that contains the values of slit, clay, sand present in that field. All these inputs are added in the database and are updated periodically. These inputs are added from a file.

2. Weather Inputs: It contains maximum and minimum temperature during the month and it also considers the value of average rainfall to balance the amount of water supplied to the crops due to irrigation and rainfall. It also takes into account daily global solar radiations.

3. Crop Inputs: It includes name of crop, crop duration and other details of the crop. It can be provided by the user.

The minimum requirements to the model can be defined as name of the crop and user can find related outputs. The requirements for checking the results for crop diversification are defined as follows:

1. The size of land is required as it is directly proportional to the production.

2. Labour and working capital is other important aspect. Labour can lead to success of crop diversification technique.

3. Yield and revenue information is also required to check outcome of the crops.[12]

The MUSTARD Model made for only mustard crop is calibrated with the models like PNUTGRO, SOYGRO from the CROPGRO Model. The dynamic crop simulation models are those that require daily or periodically update and are based on the crop phenology (various states of crop in its lifetime like emergence of leaves and flowers, development, growth, senescence, photosynthesis, respiration), infiltration, drainage and weather conditions. This testing of this model considered row to row spacing, plant to plant spacing and sowing dates of the crop to get accurate results. Then sensitive analysis was also done for the crop's phenology states by taking five coefficients that are time taken between various stages ( like plant emergence and flower appearance, first flower and first pod, first flower and first seed, first seed and physiological maturity, first flower and end of leaf expansion).[13]

\section{METHODS AND RESULTS}

The output can be calculated by using various methods. Crop Diversification can be calculated as using Simpsons Diversification Index or many other methods like Hefindahi Index or Ogive Index.

$$
\text { Simpsons Index }=1-\sum\left(\mathrm{p}_{\mathrm{i}}-\sum \mathrm{p}_{\mathrm{i}}\right)^{2}
$$

Where $p_{i}$ is the area under ith crop. The value of Simpsons Index always lies between 0 and 1 . When we have 0 it indicates completely specific crop that means no diversification and when it is 1 it indicates complete diversification.

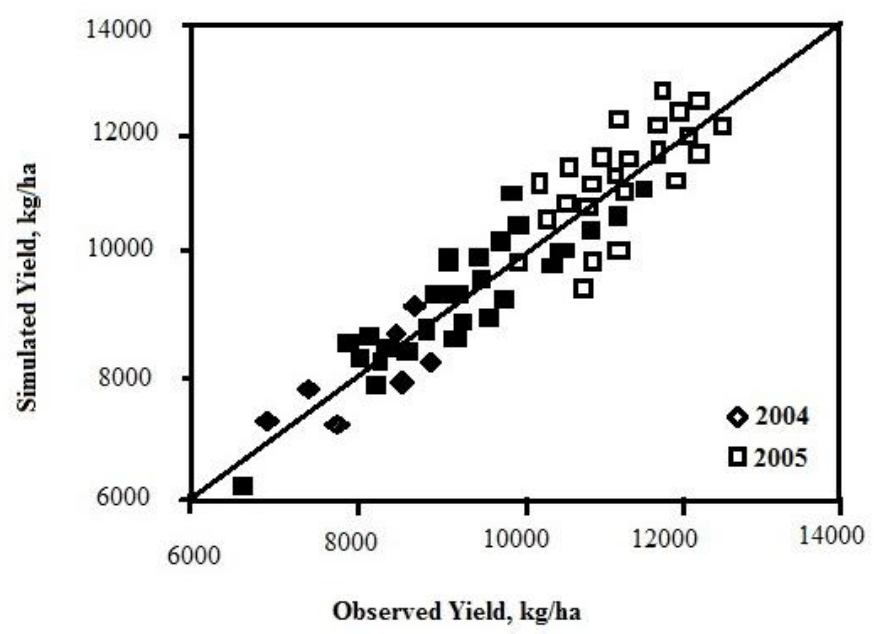

Figure 2: The simulated and observed yield of selected crop of year 2004 and 2005.

Leaf Area Index is the measure of leaf material in ecosystem and it is helpful in controlling the processes like respiration, photosynthesis and rain interception etc. It is dimensionless quantity. It can be stated as half of the total leaf area per unit ground area. It can be calculated using direct method which contains two steps. The first one is leaf collection which includes collection of waste leafs from the ground and all the leaves are collected in a leaf trap (open boxes with a definite size). The second step is leaf area determination which includes a planimetric approach that is based on the correlation between the individual leaf area and the number of area units covered by the leaf in a horizontal plain field. [14] 


\section{IJARCCE}

\section{International Journal of Advanced Research in Computer and Communication Engineering}

ISO 3297:2007 Certified

Vol. 6, Issue 6, June 2017

\section{CONCLUSION}

The evaluation of model indicates the comparison of the model outputs with the actual outputs. Thus we can see that the simulated result is mostly similar and somewhat better than the observed yield of the crops. In this model we have considered wheat, maize, rice, sugarcane, potato crops. We need the data sets that have minimum information to run the model and it should have proper information for the proper functioning of the model. The designed model is validated by comparing its simulated results with actual results that we get from the crop sown in the fields on the basis of soil and weather parameters.

\section{REFERENCES}

[1] A. Mukherjee, "Evaluation of the Policy of Crop Diversification as a Strategy for Reduction of Rural Poverty in India."

[2] C. Makate, R. Wang, M. Makate, and N. Mango, "Crop diversification and livelihoods of smallholder farmers in Zimbabwe: adaptive management for environmental change," Springerplus, vol. 5, no. 1, p. 1135, 2016.

[3] A. Chhatre, S. Devalkar, and S. Seshadri, "Crop diversification and risk management in Indian agriculture,” Decision, vol. 43, no. 2, pp. 167$179,2016$.

[4] G. Schroth and F. Ruf, "Farmer strategies for tree crop diversification in the humid tropics. A review," Agron. Sustain. Dev., vol. 34, no. 1, pp. 139-154, 2014.

[5] M. Reckling et al., “A cropping system assessment framework???Evaluating effects of introducing legumes into crop rotations,” Eur. J. Agron., vol. 76, pp. 186-197, 2016.

[6] H. A. Webber, C. A. Madramootoo, M. Bourgault, M. G. Horst, G. Stulina, and D. L. Smith, "Adapting the CROPGRO model for saline soils: The case for a common bean crop," Irrig. Sci., vol. 28, no. 4, pp. 317-329, 2010.

[7] N. Sharma and S. P. Singh, "Agricultural Diversification in Indian Punjab: An Assessment of Government Intervention Through Contract Farming,” J. Agric. Food Inf., vol. 15, no. 3, pp. 191-213, 2014.

[8] R. Sarker and T. Ray, “An improved evolutionary algorithm for solving multi-objective crop planning models," Comput. Electron. Agric., vol. 68, no. 2, pp. 191-199, 2009.

[9] L. Mahy, B. E. T. I. Dupeux, G. Van Huylenbroeck, and J. Buysse, "Simulating farm level response to crop diversification policy," Land use policy, vol. 45, pp. 36-42, 2015.

[10] H. Q. Nguyen, “Analyzing the economies of crop diversification in rural Vietnam using an input distance function," Agric. Syst., vol. 153, pp. $148-156,2017$

[11] B. Bradshaw, H. Dolan, and B. Smit, "Farm-level adaptation to climatic variability and change: Crop diversification in the Canadian prairies," Clim. Change, vol. 67, no. 1, pp. 119-141, 2004

[12] J. W. Jones et al., The DSSAT cropping system model, vol. 18, no. 3-4. 2003.

[13] P. Kaur, H. Singh, P. K. Kingra, and J. Mukherjee, "Mustard model as an agronomic management tool for oilseed Brassica cultivation in Irrigated plains of Punjab state," vol. 3, no. 2, pp. 99-110, 2012.

[14] B. Duchemin, P. Maisongrande, G. Boulet, and I. Benhadj, "A simple algorithm for yield estimates: Evaluation for semi-arid irrigated winter wheat monitored with green leaf area index," Environ. Model. Softw., vol. 23, no. 7, pp. 876-892, 2008. 